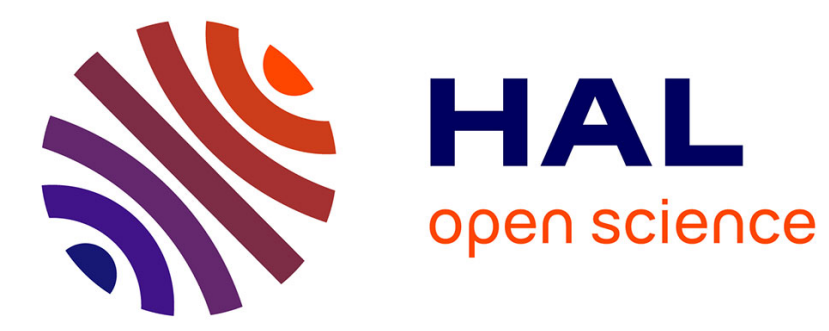

\title{
Isotropic Chiral Acoustic Phonons in 3D Quasicrystalline Metamaterials
}

Yi Chen, Muamer Kadic, Sébastien Guenneau, Martin Wegener

\section{To cite this version:}

Yi Chen, Muamer Kadic, Sébastien Guenneau, Martin Wegener. Isotropic Chiral Acoustic Phonons in 3D Quasicrystalline Metamaterials. Physical Review Letters, 2020, 124 (23), pp.235502 (6). hal02993964

\section{HAL Id: hal-02993964 \\ https://hal.science/hal-02993964}

Submitted on 7 Nov 2020

HAL is a multi-disciplinary open access archive for the deposit and dissemination of scientific research documents, whether they are published or not. The documents may come from teaching and research institutions in France or abroad, or from public or private research centers.
L'archive ouverte pluridisciplinaire HAL, est destinée au dépôt et à la diffusion de documents scientifiques de niveau recherche, publiés ou non, émanant des établissements d'enseignement et de recherche français ou étrangers, des laboratoires publics ou privés. 


\title{
Isotropic chiral acoustic phonons in $3 \mathrm{D}$ quasi-crystalline metamaterials
}

\author{
Yi Chen ${ }^{1}$ 米 Muamer Kadic ${ }^{2}$, Sébastien Guenneau ${ }^{3}$, and Martin Wegener ${ }^{1,4}$ \\ ${ }^{1}$ Institute of Applied Physics, Karlsruhe Institute of Technology (KIT), 76128 Karlsruhe, Germany \\ ${ }^{2}$ Institut FEMTO-ST, UMR 6174, CNRS, Université de Bourgogne Franche-Comté, 25000 Besançon, France \\ ${ }^{3}$ UMI 2004 Abraham de Moivre-CNRS, Imperial College London, London SWr 2AZ, United Kingdom and \\ ${ }^{4}$ Institute of Nanotechnology, Karlsruhe Institute of Technology (KIT), 76021 Karlsruhe, Germany
}

(Dated: May 2, 2020)

\begin{abstract}
The elastic properties of three-dimensional (3D) crystalline mechanical metamaterials, unlike those of amorphous structures, are generally strongly anisotropic - even in the long-wavelength limit and for highly symmetric crystals. Aiming at isotropic linear elastic wave propagation, we therefore study 3D periodic approximants of 3D icosahedral quasi-crystalline mechanical metamaterials consisting of uniaxial chiral meta-rods. Considering increasing order of the approximants, we approach nearly isotropic effective speeds of sound and isotropic acoustical activity. The latter is directly connected to circularly polarized 3D metamaterial chiral acoustic phonons - for all propagation directions in three dimensions.
\end{abstract}

In aiming at effective-medium properties that go beyond those of their ingredient materials, disordered and crystalline rationally designed composites called metamaterials have been investigated extensively throughout recent years [1], including optical [2] 6], transport [1], and mechanical properties [7-14]. Often, three-dimensional isotropic effective material properties are desirable in applications. For instance, cubic crystal symmetry guarantees isotropic behavior for electric conduction, particle diffusion, thermal conduction, thermal expansion, and the long-wavelength limit of optics [15]. This statement is not valid though for transverse elastic waves in mechanics, for which cubic and other crystal symmetries generally lead to very highly anisotropic effective properties, even in the strict long-wavelength limit 15.19.

Concerning isotropic elastic properties, this situation leaves one with the possibilities of disordered, extraordinary crystalline, and quasi-crystalline architectures. So far, certain isotropic elastic properties have been realized in achiral disordered foams [20, which are effectively isotropic on average, and in achiral extraordinary crystals designed by topology optimization 21. Following the discovery of atomic 3D quasi-crystals 22 28, isotropic achiral elastic properties have also been obtained in 3D quasi-crystals 23, 29. However, isotropic elastic properties related to chirality have not been demonstrated in any system by any means so far.

Chiral mechanical metamaterials have emerged recently, but their properties have been highly anisotropic 18, 30, 31. Acoustical activity 32 34 - the mechanical counterpart of the well-known phenomenon of optical activity [35] - is a paradigm. Here, chiral phonons instead of the textbook linearly polarized transverse acoustic (TA) phonons are the eigenstates. An incident linear phonon polarization hence rotates during phonon propagation. Therefore, an application of acoustical activity is polarization mode conversion from one transverse linear polarization to another transverse one, e.g., to the orthogonal transverse one. However, in cubic metamaterial crystals, acoustical activity has been restricted to special propagation directions deviating from the principal cubic axes by no more than just a few degrees [19, 36, and yet smaller solid angles around the cubic space diagonals. The underlying reason is fundamental: Acoustical activity is directly connected to circular eigenpolarizations of the two lowest-frequency acoustic-phonon branches, that is, to chiral phonons. To obtain circular polarization, the axis of the phonon wave vector must at least exhibit three-fold rotational symmetry [37] - locally, or at least on average. Furthermore, as the effects of chirality generally tend to zero in the strict limit of large samples ("bulk") and long wavelengths [19, 36, one must depart from the strict long-wavelength limit to obtain appreciable effects of acoustical activity in the bulk.

In this Letter, we investigate the possibility of obtaining isotropic effective elastic behavior related to chirality by introducing 3D quasi-crystalline mechanical metamaterials.

Our rational design strategy starts with the cut-andproject method [25, 27, 38, applied to a $6 \mathrm{D}$ simple-cubic lattice of points with lattice constant $a_{6 \mathrm{D}}$. The projection matrix

$$
\mathbf{M}=\frac{1}{\sqrt{1+\tau_{0}^{2}}}\left(\begin{array}{cccccc}
\tau_{1} & \tau_{1} & 0 & -1 & 0 & 1 \\
0 & 0 & 1 & \tau_{1} & 1 & \tau_{1} \\
1 & -1 & -\tau_{1} & 0 & \tau_{1} & 0 \\
\tau_{2} & -\tau_{2} & 1 & 0 & -1 & 0 \\
-1 & -1 & 0 & -\tau_{2} & 0 & \tau_{2} \\
0 & 0 & \tau_{2} & -1 & \tau_{2} & -1
\end{array}\right)
$$

acts onto a six-dimensional coordinate vector. The resulting first three components represent the lattice points in 3D physical space, while the other three components represent a fictitious vector in internal space. According to the cut-and-project method for quasi-crystals 27 and periodic approximants 25] (see Fig. S1 41]), not all of the resulting points in 3D physical space are accepted as lattice points of the 3D quasi-crystal lattice. Rather, only the points for which the internal vectors 
lie in a certain acceptance domain are used (cf. upper row of Fig. S2 [41]). The projection of the Wigner-Seitz cell of the 6D simple-cubic lattice onto the internal space defines this acceptance domain. We also include those lattice points that lie exactly on the surface of the acceptance domain, which, following [25], lie inside of the acceptance domain if the acceptance domain is shifted by an infinitesimal amount along the body diagonal direction. If the nonzero entries of the matrix in Eq. (1) are chosen to be $\tau_{1}=\tau_{2}=\tau_{0}$, with the golden ratio $\tau_{0}=(1+\sqrt{5}) / 2$, we obtain a 3D icosahedral quasi-crystal. If, instead, we set $\tau_{1}=\tau_{0}$ and $\tau_{2}$ to be a rational approximation of the golden ratio, $\tau_{2}=q / p=F_{n+1} / F_{n}$ with the elements of the Fibonacci series $F_{n}=1,1,2,3,5,8, \ldots$, we obtain a $3 \mathrm{D}$ crystal. Its three simple-cubic lattice vectors are $\left(a_{q / p}, 0,0\right),\left(0, a_{q / p}, 0\right)$ and $\left(0,0, a_{q / p}\right)$, with the lattice constant $a_{q / p}=2 a_{6 \mathrm{D}}\left(q \tau_{0}+p\right) / \sqrt{1+\tau_{0}^{2}}$. For fractions $q / p$ approaching $\tau_{0}$, the $3 \mathrm{D}$ periodic cell becomes increasingly large and the corresponding 3D crystal approaches a $3 \mathrm{D}$ icosahedral quasi-crystal. Considering the crystalline approximants is important here because it allows to apply Floquet-Bloch's theorem for the calculation of the phonon band structures. Alternatively, one can possibly solve the eigenvalue problem of an exact quasicrystal lattice by applying Floquet-Bloch's theorem in a higher dimension, and then obtain the band structure through a similar cut-and-project method [26]. Below, we will use $q / p=1 / 1,2 / 1,3 / 2$, and $5 / 3$. The corresponding architectures are illustrated in Fig. S2 [41].

To arrive at a practical physical structure, we connect the fictitious points derived from the previous paragraph by ordinary elastic (that is, achiral) homogeneous cylindrical rods in such a way that we obtain nearly isotropic phonon dispersion relations and a negligibly small relative frequency splitting between the lowest two bands in the extended zone scheme for all phonon wave vectors. This aspect is crucial because the frequency splitting between left- and right-handed circularly polarized transverse modes induced by chirality competes with any unwanted effective anisotropy for the achiral case that also lifts the degeneracy between the two orthogonally polarized TA branches. We apply two rules. First, two lattice points are connected if the corresponding $6 \mathrm{D}$ lattice points have been nearest neighbors. The resulting architecture can be seen as being composed of two types of rhombohedra, a thinner and a thicker one (see zoom-in in Fig. 1(a)). This 3D arrangement of touching rhombohedra is analogous to the 2D aperiodic Penrose tilings [39. All edges of the rhombohedra have same length (red in the inset of the Fig. 1(a)). Second, we additionally introduce segments along the face diagonals (blue dotted) of both types of rhombohedra and along the shortest body diagonal (dashed gray; only one such segment occurs in the inset of Fig. 1(a)) for the thinner rhombohedra. By increasing the local coordination num-

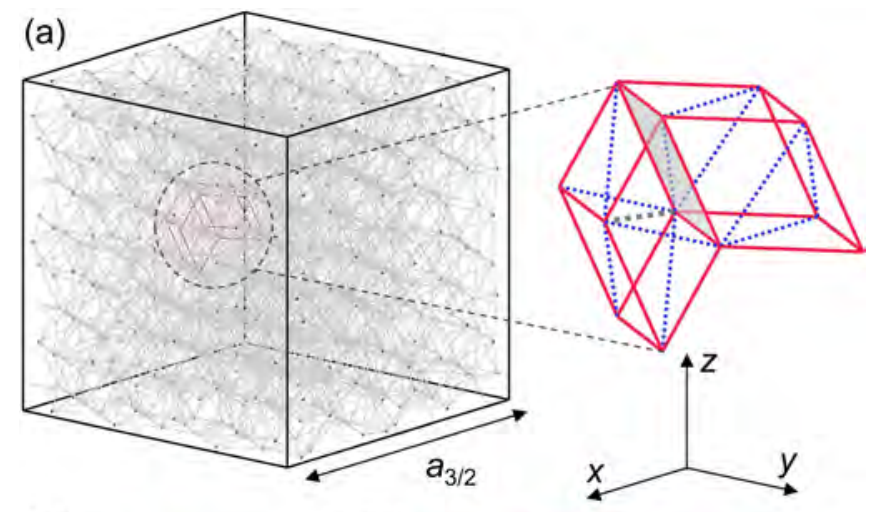

(b)

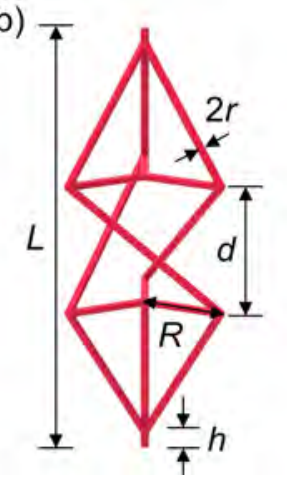

(c)

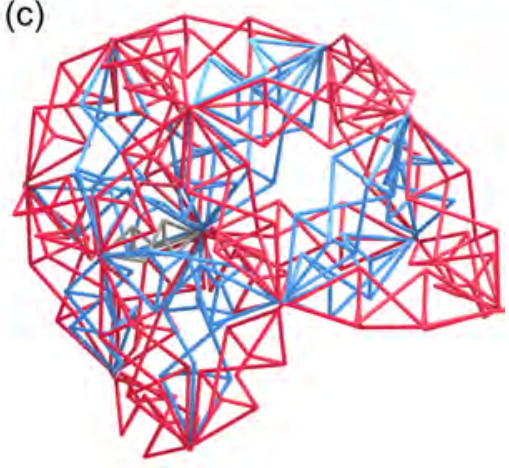

FIG. 1. (a) Illustration of the $q / p=3 / 2$ approximant of a 3D icosahedral quasi-crystalline metamaterial. The black points result from the cut-and-project method. The zoomin highlights three types of segments between these points, i.e., the edges (red), the face diagonals (blue dotted) of the two types of rhombohedra, and the shortest body diagonals (gray dashed) of the thinner rhombohedra. An animated version of the 3/2 approximant is shown in Supplemental Video 1. If we replace each segment by either an achiral homogeneous cylindrical rod or by a homogeneous uniaxial chiral rod, we approach an achiral or a chiral 3D quasi-crystal in the limit of large approximant order, respectively. (b) Next, we approximate a homogeneous uniaxial chiral rod with full rotational symmetry by the depicted chiral "meta-rod" with three-fold rotational symmetry. The meta-rod is composed of ordinary achiral elastic rods. This approximation is justified for wavelengths large compared to the length $L$. (c) Resulting chiral architecture corresponding to the zoomed-in region in (a). Throughout this Letter, we consider the following normalized parameters: $d / L=0.3, R / L=0.2, h / L=0.035$, $r / a_{6 \mathrm{D}}=0.01$ (leading to the same rod radius for all rods in the architecture for fixed $a_{6 \mathrm{D}}$ ), and fixed $a_{6 \mathrm{D}}=100 \mu \mathrm{m}$, which is amenable to state-of-the-art 3D laser printing of polymers 18. Therefore, we use typical polymer parameters for all rods: Young's modulus $E=4.18 \mathrm{GPa}$, Poisson's ratio $\nu=0.4$, and mass density $\rho=1.15 \times 10^{3} \mathrm{~kg} / \mathrm{m}^{3}$.

ber, the face-diagonal segments play an important role in decreasing the anisotropy-induced splitting between the two transverse bands along the principal cubic directions. Likewise, the mentioned body diagonals are critical to decrease the anisotropy-induced splitting for wave vectors along the face diagonals of the $3 \mathrm{D}$ simple-cubic 
translational lattice. Without these two additional types of segments, the properties of the metamaterial converge more slowly towards the isotropic case with increasing approximant order. The mentioned three types of links are highlighted by the red, dashed blue, and dashed gray segments in Fig. 1(a). Their respective lengths are $a_{6 \mathrm{D}}$, $a_{6 \mathrm{D}} 2 / \sqrt{1+\tau_{0}^{2}}$, and $a_{6 \mathrm{D}} \sqrt{6-3 \tau_{0}} / \sqrt{1+\tau_{0}^{2}}$. Finally, each segment is replaced by a cylindrical rod composed of an ordinary achiral elastic material. Calculated band structures and contours for these achiral approximants of 3D quasi-crystals with rod radius $r / a_{6 \mathrm{D}}=0.01$ are depicted in Fig. S3 and Fig. S4 [41, respectively. The discussed procedure leads to isotropic achiral properties for increasing approximant order.

To achieve chiral metamaterial architectures supporting isotropic chiral phonons, we can conceptually replace each segment of the previous section (i.e., the red, dotted blue, and dashed gray segments in Fig. 1(a)) by a homogeneous uniaxial chiral rod to again obtain an approximant of a true 3D quasi-crystal. Unfortunately, rods made of a homogeneous ordinary material with chiral properties do not exist in reality. Therefore, we approximate and replace each rod by the chiral "meta-rod" shown in Fig. 1(b). This meta-rod is composed of ordinary homogeneous achiral elastic rods. Its length, $L$, is scaled according to the segment length. In the limit of vanishing $R$, the meta-rod becomes achiral. In the limit of large $R$, neighboring meta-rods overlap. Our choice is a trade-off. By virtue of the three-fold rotational symmetry of the meta-rod, and independent on its randomly chosen azimuthal angle (see [41), its effective properties in the limit that the wavelength is large compared to $L$ are those of a homogeneous uniaxial chiral rod, supporting chiral phonons propagating along the rod axis. Purely geometrically speaking, the truss lattice itself ( $c f$. Fig. 1(c)) does not converge to a 3D quasi-crystal.

For the $q / p=1 / 1,2 / 1,3 / 2$, and $5 / 3$ approximants considered below, this procedure leads to $32,136,576$, and 2240 points within one periodic cell, respectively, and to $228,920,3398$, and 16768 chiral meta-rods within one periodic cell, respectively. To deal with periodic cells containing so many rods, we treat all rods by using Timoshenko-beam theory and COMSOL Multiphysics ${ }^{\circledR}$ (MUMPS solver) [40]. This approximation is justified because the rods are very slender $\left(r / a_{6 \mathrm{D}}=0.01\right.$ in Fig. 1(b)). For the meta-rod shown in Fig. 1(b), a direct comparison to continuum mechanics is provided in Fig. S5 [41].

In Figs. 2(a)-(d), we plot the calculated phonon dispersion relations along cubic face diagonals and principal cubic directions for the $q / p=1 / 1,2 / 1,3 / 2$, and $5 / 3$ approximants. The lowest two transverse phonon branches and their back-folded parts are colored in red. The other, less important, bands are colored in gray. A frequency splitting between the two red transverse bands is clearly visible for both propagation di-

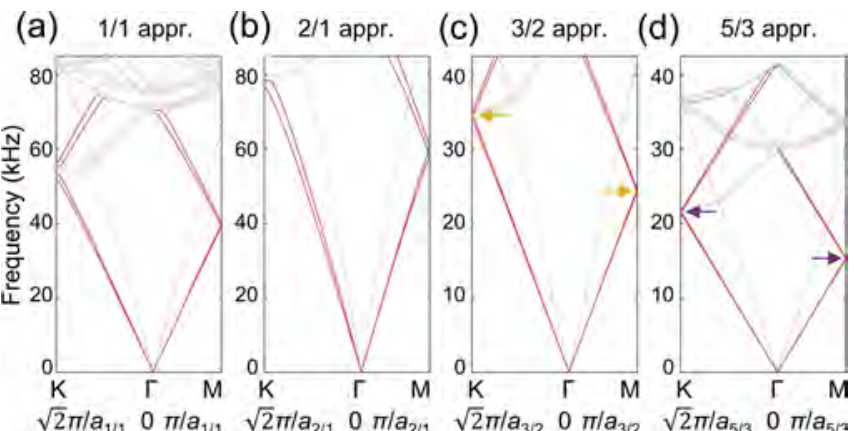

(e)

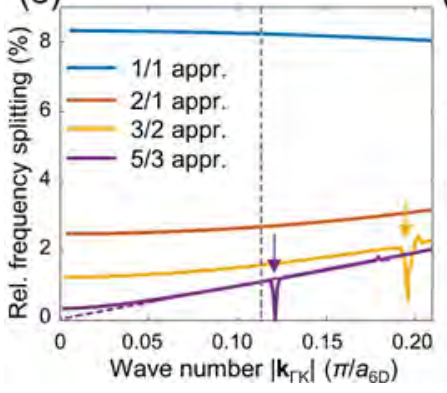

(f)

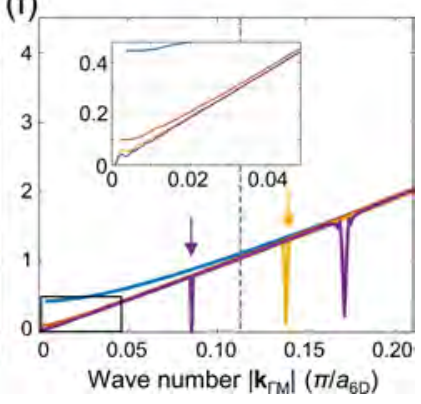

FIG. 2. Calculated phonon dispersion relations for approximant architectures as illustrated in Fig. 1 for propagation along a cubic face diagonal ( $\Gamma \mathrm{K}$ direction, left) and a principal cubic direction ( $\Gamma \mathrm{M}$ direction, right). (a) $q / p=1 / 1$, (b) $q / p=2 / 1$, (c) $q / p=3 / 2$, and (d) $q / p=5 / 3$. The lowest two transverse bands and their back-folded parts are colored in red, the other less important bands are plotted in gray. These data are shown in magnified form in Fig. S6 41. (e) and (f) exhibit the relative frequency splitting, $2\left(f_{2}-f_{1}\right) /\left(f_{2}+f_{1}\right)$, between the two transverse bands. The colors for the four approximants are indicated in the legend. (e) $\Gamma K$ direction. (f) $\Gamma \mathrm{M}$ direction. The results shown in Figs. 3 and 4 have been evaluated for the wave number indicated by the dashed gray straight lines.

rections. This splitting can generally have two different origins: Unwanted anisotropy and wanted chirality. The anisotropy results in linearly or elliptically polarized eigenmodes, whereas chirality alone ideally leads to chiral phonons connected to circularly polarized eigenmodes. In the long-wavelength limit of a periodic structure, the anisotropy-induced relative frequency splitting is independent of the wave number $|\mathbf{k}|$. In contrast, the chirality-induced splitting should vanish $\propto|\mathrm{k}|$ in the long-wavelength limit, for which classical Cauchy elasticity applies [15]. Therefore, we expect the splitting at $|\mathbf{k}|=0$ to be anisotropy-induced only, which is unwanted. It is thus instructive to plot the relative frequency splitting, $2\left(f_{2}-f_{1}\right) /\left(f_{2}+f_{1}\right)$, versus the phonon wave number $|\mathbf{k}|$ in Figs. 2(e) and (f). The dips in the curves indicated by the arrows occur on the edge of the first Brillouin zone for the corresponding approximant periodic cell. Clearly, the relative frequency splitting at $|\mathbf{k}|=0$ converges to zero with increasing approximant order (see Figs. 2(e) and (f)). For the highest approximant order numerically 


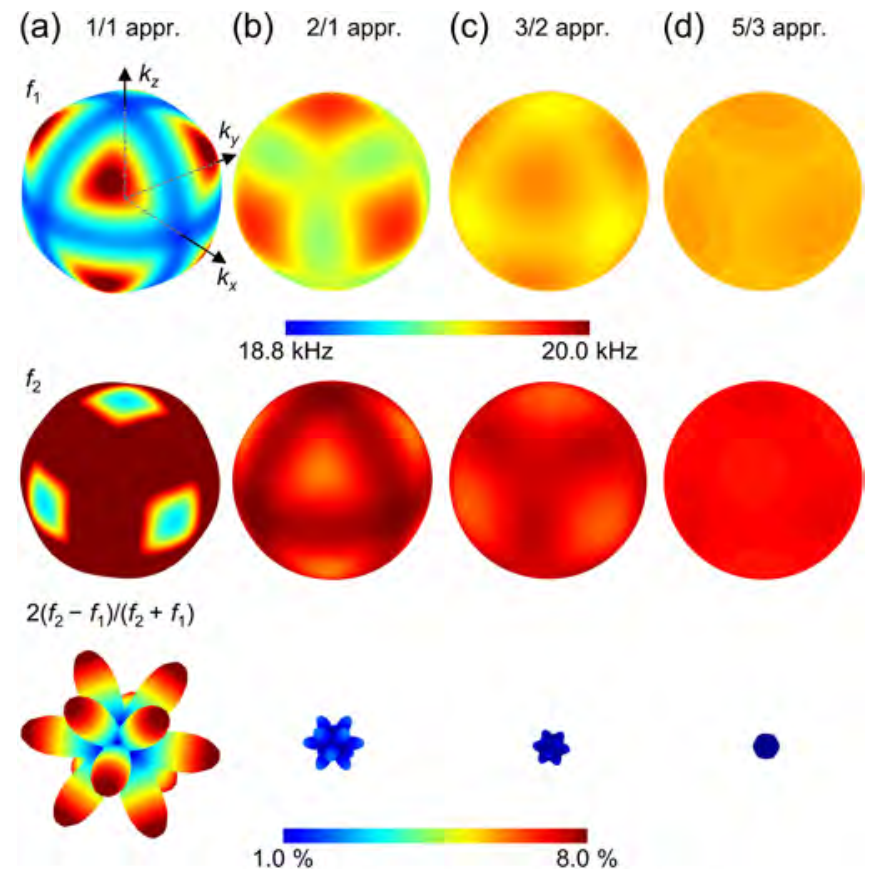

FIG. 3. Calculated results for increasing order (from left to right) of $3 \mathrm{D}$ quasi-crystal approximants for $|\mathbf{k}|=1 / 9\left(\pi / a_{6 \mathrm{D}}\right)$ (cf. dashed gray lines in Figs. 2(e) and (f)). (a) $q / p=1 / 1$, (b) $q / p=2 / 1$, (c) $q / p=3 / 2$, and (d) $q / p=5 / 3$. In the first two rows, we depict the direction dependence of the two transverse phonon eigenfrequencies, $f_{1}$ and $f_{2}$ on a false-color scale. In addition, the length of the vector from the origin to the surface of the plot is proportional to the eigenfrequency for this propagation direction. In the third row, we depict the relative frequency splitting between the two transverse eigenfrequencies, $2\left(f_{2}-f_{1}\right) /\left(f_{2}+f_{1}\right)$, in the same manner.

accessible to us, i.e., $q / p=5 / 3$, the relative splitting for $|\mathbf{k}|=0$ is about $0.3 \%$ for wave vectors along the cubic face diagonals (see Fig. 2(e)) and less than $0.03 \%$ for the principal cubic directions (see Fig. 2(f)). As expected from micropolar continuum elasticity [19], the chiralityinduced relative splitting converges towards a behavior $\propto|\mathbf{k}|$ (see the purple dashed straight line in Fig. 2(e)). At the indicated gray line at $|\mathbf{k}|=1 / 9\left(\pi / a_{6 \mathrm{D}}\right)$, chiralityinduced splitting dominates over the anisotropy-induced splitting and we expect chiral phonon polarizations for the two sets of directions considered. Indeed, inspection of the corresponding eigenmodes reveals circular phonon polarization (see Fig. S7 and Supplemental Videos 2 and 3 [41]).

Yet more importantly, based on our entire design process, we expect an isotropic chiral behavior of the acoustic phonons in the limit of increasing approximant order. To numerically test this conjecture, we visualize the three-dimensional direction dependence of the eigenfrequencies $f_{1}$ and $f_{2}$ in Fig. 3 and of the eigenmode polarizations in Fig. 4 (again for a fixed modulus of the phonon wave vector of $\left.|\mathbf{k}|=1 / 9\left(\pi / a_{6 \mathrm{D}}\right)\right)$. For the $q / p=5 / 3$ approximant in Fig. 3 , the frequency $f_{1}$ varies only between a minimum of $19.61 \mathrm{kHz}$ and a maximum of $19.66 \mathrm{kHz} . f_{2}$ varies between $19.82 \mathrm{kHz}$ and $19.86 \mathrm{kHz}$. The corresponding relative frequency splitting $2\left(f_{2}-f_{1}\right) /\left(f_{2}+f_{1}\right) \approx 1 \%$ is nearly isotropic, too. In the ideal isotropic limit, $f_{1}$, $f_{2}$, and $2\left(f_{2}-f_{1}\right) /\left(f_{2}+f_{1}\right)$ should be constant. In Fig. 4 , we visualize the eigenpolarizations of the two transverse phonon bands. This is accomplished in two different ways. First, we define the polarization degree as the ratio, $\zeta$, of the minor and major axes of the polarization ellipse formed by the mean displacement vector of the periodic cell versus time: $\zeta=1$ corresponds to circular polarization, $\zeta=0$ to linear polarization, and values in between to elliptical polarization. In the top row of Fig. 4, we false-color code $\zeta$ onto the surface of a sphere in $3 \mathrm{D}$ wave vector space. From this representation, one can see that the ideal of constant $\zeta=1$ is approached with increasing approximant order. However, one cannot see the orientation of the polarization ellipse. Therefore, we depict in the bottom row of Fig. 4 the real-space trace of the mean displacement vector, again for the two transverse bands, $i=1,2$. Obviously, very nearly circular polarization is achieved for all wave vector directions for the highest $q / p=5 / 3$ approximant, whereas linear or elliptical polarizations still dominate for the $1 / 1$ and the $2 / 1$ approximants. If the local coordination number is reduced by considering only the red connections in Fig. 1(a), the isotropic ideal is not yet reached for the 5/3 approximant (cf. Fig. S8 41]).

In conclusion, we have presented a rational design of truss-based chiral 3D mechanical-metamaterials exhibiting nearly isotropic chiral acoustic phonons (and as a special limit also isotropic achiral acoustic phonons). Our design approach is based on 3D quasi-crystal approximants of increasing order. Intuitively, for sufficiently large wavelengths and on average over the course of propagation through the infinite 3D periodic approximant, the elastic wave "feels" an isotropic chiral medium with complete rotational symmetry around any wave propagation direction. This averaging should make the architecture robust against disorder but comes at the price of reduced chiral effects. Here, we achieve an isotropic relative frequency splitting of $1 \%$, which compares to maxima over $10 \%$ in highly anisotropic cubic 3D metamaterial crystals 31. Future experimental realizations of our proposal appear possible based on advanced 3D additive manufacturing. However, the more than ten thousand chiral meta-rods per 3D approximant periodic cell pose a formidable challenge.

Y.C. acknowledges support by the Alexander von Humboldt foundation and by the National Natural Science Foundation of China (contract 11802017). This research has additionally been funded by the Deutsche Forschungsgemeinschaft (DFG, German Research Foundation) under Germany's Excellence Strategy via the Excellence Cluster "3D Matter Made to Order" (EXC2082/1-390761711), which has also been supported by 


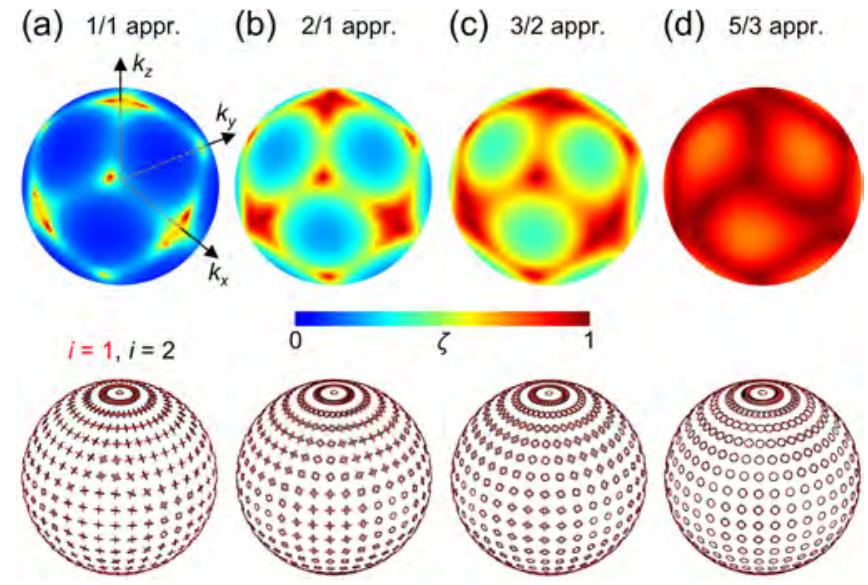

FIG. 4. Calculated results for increasing order (from left to right) of 3D quasi-crystal approximants for $|\mathbf{k}|=1 / 9\left(\pi / a_{6 \mathrm{D}}\right)$ (cf. dashed gray lines in Figs. $2(\mathrm{e})$ and (f)). (a) $q / p=1 / 1$, (b) $q / p=2 / 1$, (c) $q / p=3 / 2$, and (d) $q / p=5 / 3$. The first row exhibits the polarization degree, $\zeta$, on a false-color scale $(c f$. Fig. 3). $\zeta=0$ corresponds to linear polarization and $\zeta=1$ to circular polarization. The second row shows real-space trajectories of the phonon displacement vector versus time (mean over one periodic cell), i.e., a circle corresponds to circular phonon polarization, an ellipse to elliptical polarization, and a line to linear polarization. The center of each miniature on the sphere indicates the corresponding wave vector direction in 3D. The behavior of the two transverse phonon bands, with indices $i=1$ and $i=2$, is shown in red and black, respectively. For the $5 / 3$ approximant, chiral phonons are obtained for all phonon propagation directions in $3 \mathrm{D}$.

the Carl Zeiss Foundation through the "Carl-ZeissFoundation-Focus@HEiKA", by the State of BadenWürttemberg, and by the Karlsruhe Institute of Technology (KIT). We further acknowledge support by the Helmholtz program "Science and Technology of Nanosystems" (STN), and by the associated KIT project "Virtual Materials Design" (VIRTMAT). M.K. is grateful for support by the EIPHI Graduate School (contract ANR-17EURE-0002) and by the French Investissements d'Avenir program, project ISITEBFC (contract ANR-15-IDEX$03)$.

yi.chen@partner.kit.edu;chenyi221@gmail.com

[1] M. Kadic, G. W. Milton, M. van Hecke, and M. Wegener, Nat. Rev. Phys. 1, 198 (2019)

[2] A. DellaVilla, S. Enoch, G. Tayeb, V. Pierro, V. Galdi, and F. Capolino, Phys. Rev. Lett. 94, 183903 (2005).

[3] E. Plum, X. X. Liu, V. A. Fedotov, Y. Chen, D. P. Tsai, and N. I. Zheludev, Phys. Rev. Lett. 102, 113902 (2009)

[4] A. Alu and N. Engheta, Phys. Rev. Lett. 103, 143902 (2009).

[5] W. Cai and V. M. Shalaev, Optical metamaterials Vol. 10 (Springer, 2010).

[6] I. Fernandez-Corbaton, C. Rockstuhl, P. Ziemke,
P. Gumbsch, A. Albiez, R. Schwaiger, T. Frenzel, M. Kadic, and M. Wegener, Adv. Mater. 21, 1807742 (2019)

[7] G. Milton and R. Willis, Proc. Royal Soc. A 463, 855 (2007).

[8] X. Liu, G. Hu, G. Huang, and C. Sun, Appl. Phys. Lett. 98, 251907 (2011).

[9] R. Zhu, X. Liu, G. Hu, C. Sun, and G. Huang, Nat. Commun. 5, 5510 (2014)

[10] S. H. Kang, S. Shan, A. Košmrlj, W. L. Noorduin, S. Shian, J. C. Weaver, D. R. Clarke, and K. Bertoldi, Phys. Rev. Lett. 112, 098701 (2014)

[11] S. Krödel, N. Thomé, and C. Daraio, Extreme Mech. Lett. 4, 111 (2015)

[12] Y. Achaoui, B. Ungureanu, S. Enoch, S. Brûlé, and S. Guenneau, Extreme Mech. Lett. 8, 30 (2016).

[13] C. Coulais, C. Kettenis, and M. van Hecke, Nat. Phys. 14, 40 (2018).

[14] W. Wang, Y. Jin, W. Wang, B. Bonello, B. DjafariRouhani, and R. Fleury, Phys. Rev. B 101, 024101 (2020).

[15] A. Authier, International Tables for Crystallography Volume D: Physical properties of crystals (Springer, 2003).

[16] C. Lin, T. Fang, T. Zhang, S. Niu, G. Cheng, and Z. Shi, $\mathbf{5 4 , 8 0 3}$

[17] S. Babaee, J. Shim, J. C. Weaver, E. R. Chen, N. Patel, and K. Bertoldi, Adv. Mat. 25, 5044 (2013)

[18] T. Frenzel, M. Kadic, and M. Wegener, Science 358, 1072 (2017)

[19] Y. Chen, T. Frenzel, S. Guenneau, M. Kadic, and M. Wegener, J. Mech. Phys. Solids 137, 103877 (2020)

[20] R. Lakes, Science 235, 1038 (1987).

[21] A. Radman, X. Huang, and Y. Xie, Eng. Optimiz. 45, 1331 (2013).

[22] D. Shechtman, I. Blech, D. Gratias, and J. W. Cahn, Phys. Rev. Lett. 53, 1951 (1984).

[23] P. Bak, Phys. Rev. B 32, 5764 (1985)

[24] J. E. S. Socolar, T. C. Lubensky, and P. J. Steinhardt, Phys. Rev. B 34, 3345 (1986).

[25] J. Los, T. Janssen, and F. Gähler, J. Phys. I 3, 107 (1993)

[26] A. W. Rodriguez, A. P. McCauley, Y. Avniel, and S. G. Johnson, Phys. Rev. B 77, 104201 (2008).

[27] C. Janot, Quasicrystals: A Primer, Monographs on the physics and chemistry of materials (Oxford University Press, 2012).

[28] T. Janssen, G. Chapuis, and M. De Boissieu, Aperiodic crystals: from modulated phases to quasicrystals, Vol. 20 (Oxford University Press, 2018).

[29] P. S. Spoor, J. D. Maynard, and A. R. Kortan, Phys. Rev. Lett. 75, 3462 (1995)

[30] Z. Rueger and R. S. Lakes, Phys. Rev. Lett. 120, 065501 (2018)

[31] J. Reinbold, T. Frenzel, A. Münchinger, and M. Wegener, Materials 12, 3527 (2019).

[32] L. Whyte, Nature 182, 198 (1958)

[33] D. L. Portigal and E. Burstein, Phys. Rev. 170, 673 (1968)

[34] K. V. Bhagwat and R. Subramanian, Acta Crystallogr. Sect. A 44, 551 (1988).

[35] I. V. Lindell, A. H. Sihvola, S. A. Tretyakov, and A. J. Viitanen, Electromagnetic waves in chiral and biisotropic media (Artech House Boston, 1994).

[36] T. Frenzel, J. Köpfler, E. Jung, M. Kadic, and M. We- 
gener, Nat. Commun. 10, 3384 (2019).

[37] I. Fernandez-Corbaton, Opt. Express 21, 29885 (2013)

[38] M. Duneau and A. Katz, Phys. Rev. Lett. 54, 2688 (1985)

[39] R. Penrose, Bull. Inst. Math. Appl. 10, 266 (1974).

[40] T. J. Hughes, The Finite Element Method, (Dover Publications, 2000).
[41] See Supplemental Material at [URL will be inserted by publisher] for illustration of the generalized cutand-project method, details on the 3D chiral approximants, details of numerical procedure, justification of the Timoshenko-beam model, results of isotropic achiral lattices, examples of circularly polarized eigenmodes, and influence of coordination number. 


\title{
Supplemental Material: Isotropic chiral acoustic phonons in $3 \mathrm{D}$ quasi-crystalline metamaterials
}

\author{
Yi Chen, Muamer Kadic, Sébastien Guenneau, and Martin Wegener
}

(Dated: May 2, 2020) 


\section{CUBIC APPROXIMANTS FROM CUT-AND-PROJECT METHOD}

In our Letter, the (generalized) cut-and-project method $[25,27,38]$ is applied to a $6 \mathrm{D}$ simple-cubic lattice with lattice constant $a_{6 \mathrm{D}}$, leading to an ideal 3D quasi-crystal or a periodic approximant of an ideal 3D quasi-crystal, respectively. For illustration, as the procedure is hard to visualize or illustrate for $6 \mathrm{D}$, we here apply the same method to a $2 \mathrm{D}$ square lattice with lattice constant $a_{2 \mathrm{D}}$ shown in Fig. S1(a). This leads to the 1D quasi-crystal shown in Fig. S1(b) or to the periodic 1D quasi-crystal approximant shown in Fig. S1(c), respectively. In this $2 \mathrm{D}$ case, the $(6 \times 6)$-matrix given in Eq. (1) of the Letter is replaced by the simple $(2 \times 2)$-matrix

$$
\mathbf{M}=\frac{1}{\sqrt{1+\tau_{0}^{2}}}\left(\begin{array}{cc}
\tau_{1} & -1 \\
1 & \tau_{2}
\end{array}\right)
$$

where $\tau_{0}=(1+\sqrt{5}) / 2$ is the golden number. For the 1D quasi-crystal case shown in Fig. S1(b), we have $\tau_{1}=\tau_{2}=\tau_{0}$. In that case, the matrix product of $\mathbf{M}$ by its transpose gives the Identity matrix and so $\mathbf{M}$ is an orthogonal matrix. The square lattice of black dots from Fig. S1(a) is rotated with respect to the horizontal physical-space axis by an angle $\Theta=\tan ^{-1}\left(1 / \tau_{0}\right)$. The physical-space axis and the internal-space axis are perpendicular; they include an angle of 90 degrees. Black dots that lie within the gray acceptance domain (an infinite strip) are projected onto the horizontal physical-space axis. This gray area results from the rotated Wigner-Seitz cell (highlighted in red) of the 2D square lattice. The projected blue dots on the horizontal physical-space axis are the 1D quasi-crystal lattice points.

For the 1D quasi-crystal approximant shown in Fig. S1(c), we have $\tau_{1}=\tau_{0}$ and $\tau_{2}=$ $q / p$, where the integers $q=F_{n+1}$ and $p=F_{n}$ are successive Fibonacci numbers $F_{n}=$ $1,1,2,3,5,8, \ldots$ In the limit of $n \rightarrow \infty$, the fraction $F_{n+1} / F_{n}$ converges to the golden number $\tau_{0}$. The procedure illustrated in Fig. S1(c) is analogous to that in Fig. S1(b), except for the important difference that the physical-space axis and the internal-space axis no longer include an angle of 90 degrees. Here, the internal-space axis is rotated with respect to the original principal axis of the $2 \mathrm{D}$ square lattice by an angle $\Theta_{2}=\tan ^{-1}\left(1 / \tau_{2}\right)$. As a result of this "shear", the gray acceptance domain is rotated with respect to that in Fig. S1(b). The blue points projected onto the physical-space axis are periodic along the physical-space axis with period $a_{q / p}=a_{2 / 1}$. With increasing $n$, the ratio $\tau_{2}$ converges to $\tau_{0}$. Furthermore, 
the period $a_{q / p}$, which is indicated in Fig. S1(c), becomes larger and larger. Eventually, in the limit of $n \rightarrow \infty$, the periodic 1D quasi-crystal approximant of blue dots in Fig. S1(c) converges to the ideal 1D quasi-crystal of blue dots shown in Fig. S1(a).

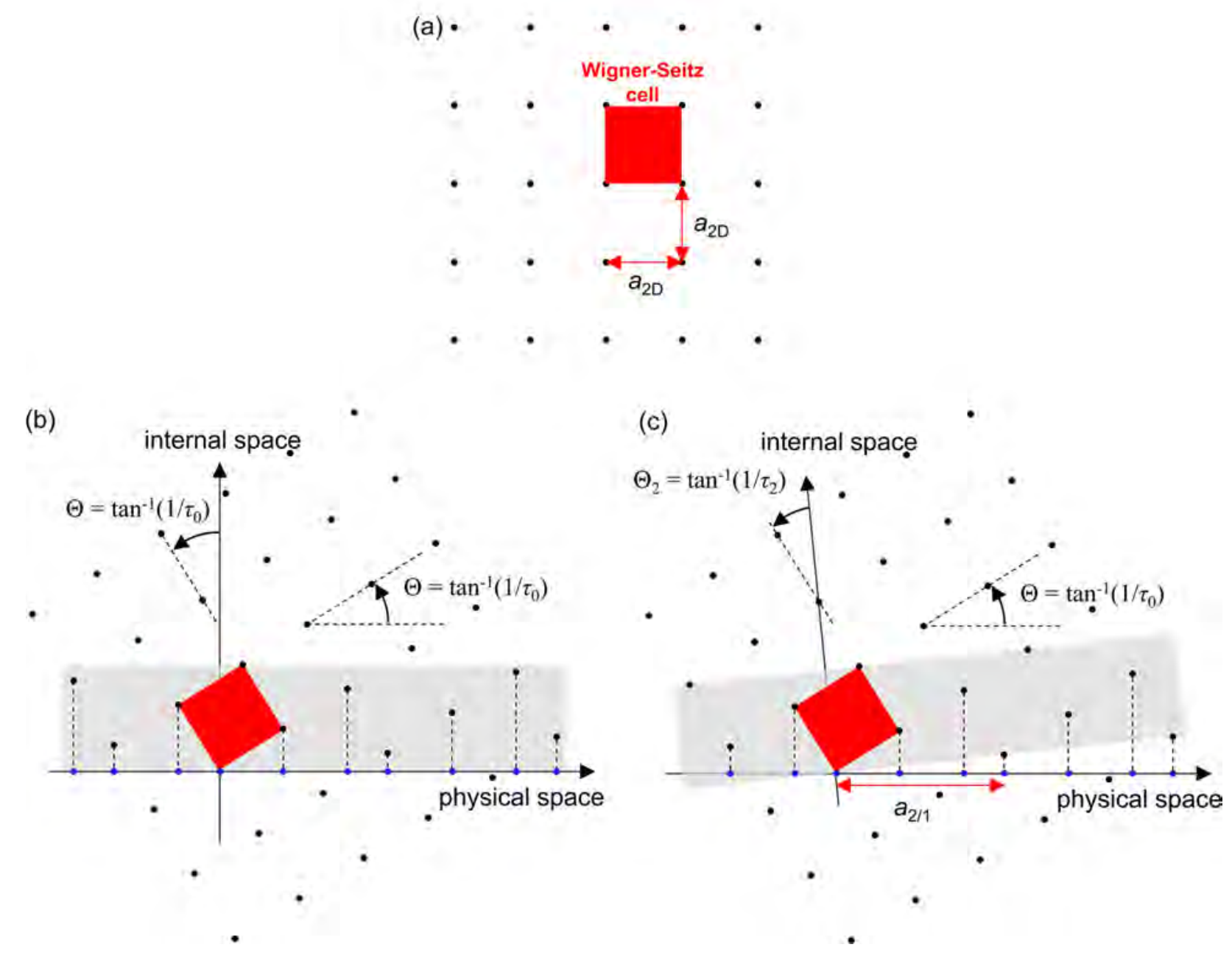

FIG. S1. Illustration of the (generalized) cut-and-project method starting from a 2D square lattice of black dots instead of the 6D simple-cubic lattice discussed in the Letter. (a) Two-dimensional square lattice of black dots with lattice constant $a_{2 \mathrm{D}}$ and its Wigner-Seitz cell (red). (b) For $\tau_{1}=\tau_{2}=\tau_{0}$, the cut-and-project method leads to an ideal 1D quasi-crystal of blue dots on the physical-space axis. (c) For $\tau_{1}=\tau_{0}$ and $\tau_{2}=q / p$ (depicted is the example of $q / p=2 / 1$ ), its generalization leads to a periodic approximant of the ideal 1D quasi-crystal of blue dots with the indicated 1D periodicity or $1 \mathrm{D}$ lattice constant $a_{q / p}$. The $1 \mathrm{D}$ acceptance domain (or interval) is highlighted by the gray area (an infinite strip). 
Fig. S2 illustrates the 3D acceptance domains (upper row) and the 3D quasi-crystal approximants (lower row) resulting from the (generalized) cut-and-project method [25, 27, 38] and the projection matrix in Eq. (1) of the Letter, starting from a 6D simple-cubic lattice with lattice constant $a_{6 \mathrm{D}}$.

(a) $\quad 1 / 1$ appr.
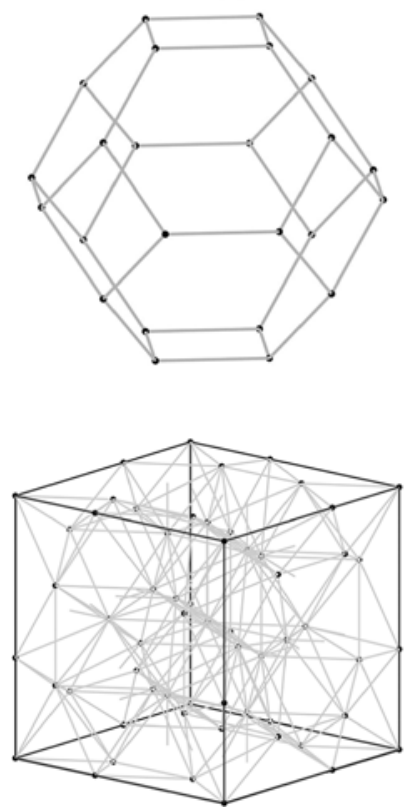

(b) 2/1 appr.
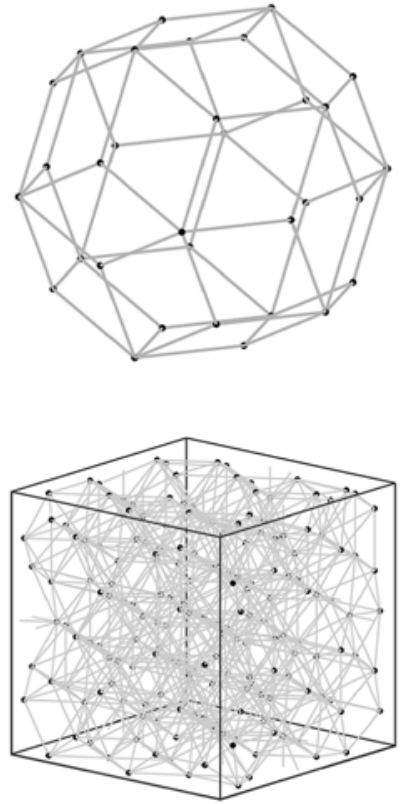

(c) $3 / 2$ appr.
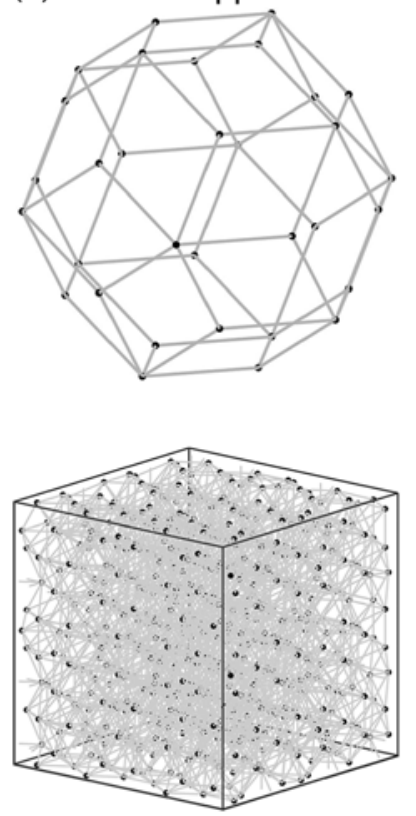

(d) $5 / 3$ appr.
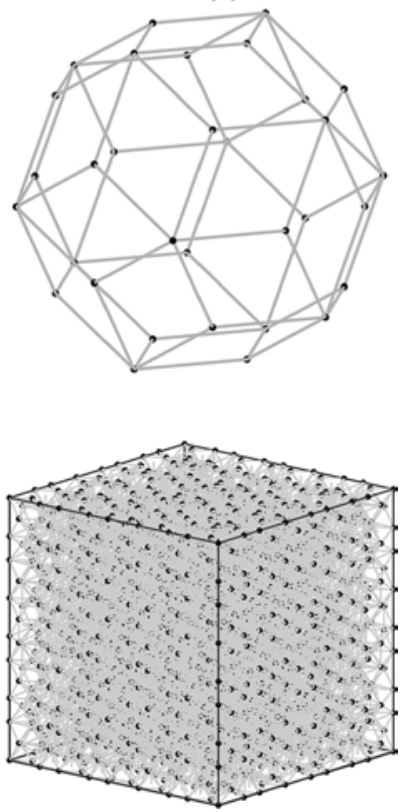

FIG. S2. Acceptance domain in internal space (upper row) and resulting corresponding approximants in physical space (lower row). (a) $q / p=1 / 1$, (b) $q / p=2 / 1$, (c) $q / p=3 / 2$, and (d) $q / p=5 / 3$. The acceptance domains converge towards the ideal quasi-crystal case. The case of $q / p=3 / 2$ has already been shown in Fig. 1(a). An animated version of the $q / p=3 / 2$ cubic approximant is given in Supplemental Video 1. 


\section{CALCULATED RESULTS FOR ACHIRAL APPROXIMANTS}

\section{Achiral approximants of icosahedral quasi-crystal lattices have been obtained by replacing}

each segment in Fig. 1(a) by a cylindrical rod (also see Fig. S2). Otherwise, the procedure, the geometrical parameters, and the numerical approach for the achiral approximants are the same as for the chiral approximants shown in the Letter, for which each segment in Fig. 1(a) is replaced by the chiral element shown in Fig.1(b). Therefore, the following discussion applies to both cases, achiral and chiral approximants. The rod radius is chosen to be $r / a_{6 \mathrm{D}}=0.01$ (also see caption of Fig. 1), with a fixed $a_{6 \mathrm{D}}=100 \mu \mathrm{m}$. For all rods, we use the Young's modulus $E=4.18 \mathrm{GPa}$, the Poisson's ratio $\nu=0.4$, and the mass density $\rho=1.15 \times 10^{3} \mathrm{~kg} / \mathrm{m}^{3}$. We solve the dynamic elasticity equation $-\rho \omega^{2} \mathbf{u}=E /(2+$ $2 \nu) /(1-2 \nu) \nabla(\nabla \cdot \mathbf{u})+E /(2+2 \nu) \nabla^{2} \mathbf{u}$ for the achiral metamaterial to obtain the band structure and corresponding eigenmodes. $\omega$ and $\mathbf{u}$ represent the angular eigenfrequency and the displacement field, respectively. We apply traction-free boundary conditions on the boundary of all rods, and apply Floquet-Bloch conditions on six sides of cubic approximants. The elasticity equation is solved using a finite-element approach implemented within the commercial software COMSOL Multiphysics ${ }^{\circledR}$ (MUMPS solver). All the rods are discretized using the Timoshenko beam module in COMSOL Multiphysics ${ }^{\circledR}$ with a mesh size smaller than $L / 10$. The equations underlying the Timoshenko beam approach are given in Section 5.4. of $[40]$. 

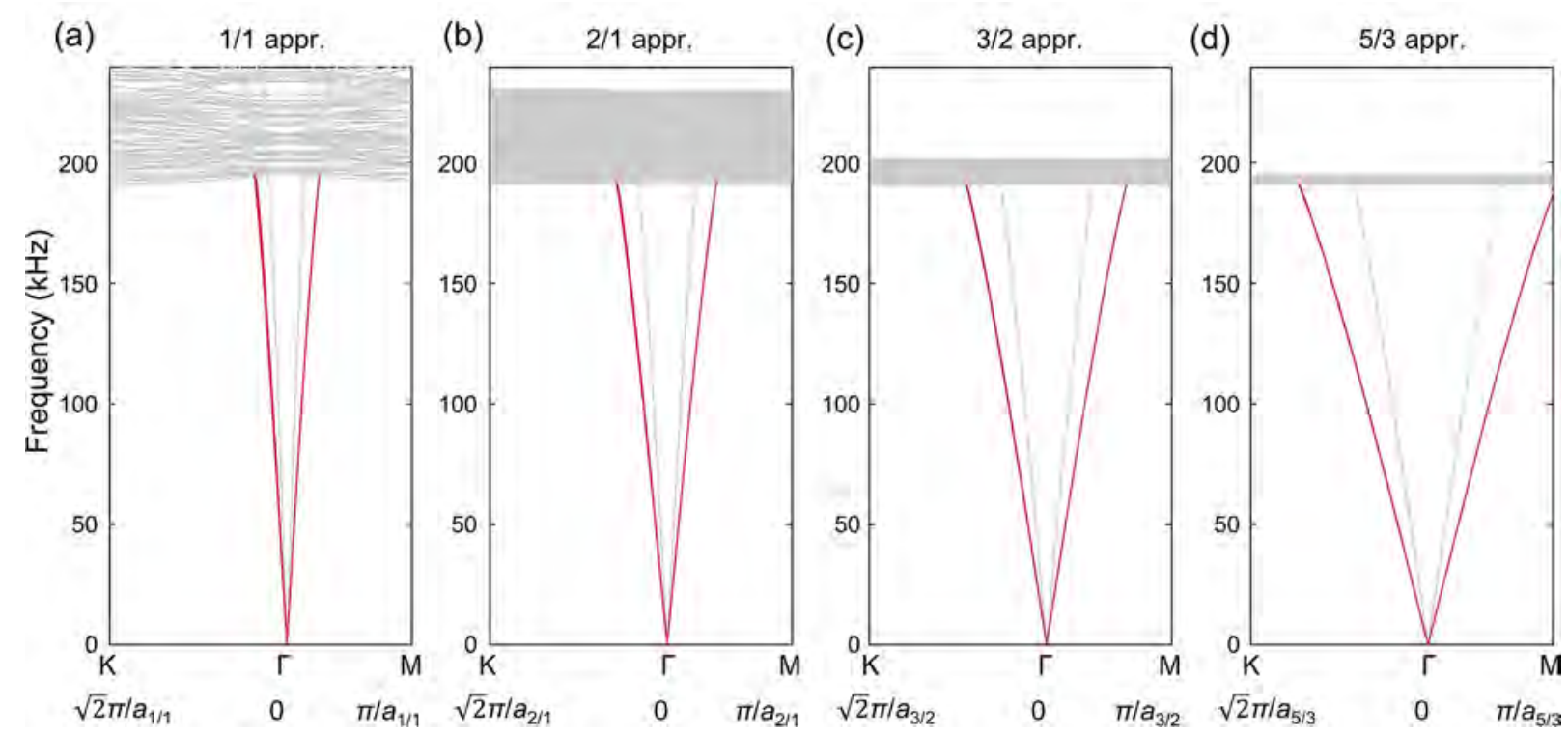

FIG. S3. Numerically calculated phonon dispersion relations along the cubic face diagonals ( $\Gamma K$ direction, left) and the principal cubic directions ( $Г \mathrm{M}$ direction, right) for achiral approximants. (a) $q / p=1 / 1$, (b) $q / p=2 / 1$, (c) $q / p=3 / 2$, and (d) $q / p=5 / 3$. The lowest two transverse bands are colored in red, the other bands are plotted in gray. Here, we have restricted ourselves to the lowest 200 bands. The gray bands can barely be separated because they lie so closely. The frequency splitting between the two transverse bands is hardly visible, which is desirable for an achiral isotropic material. 


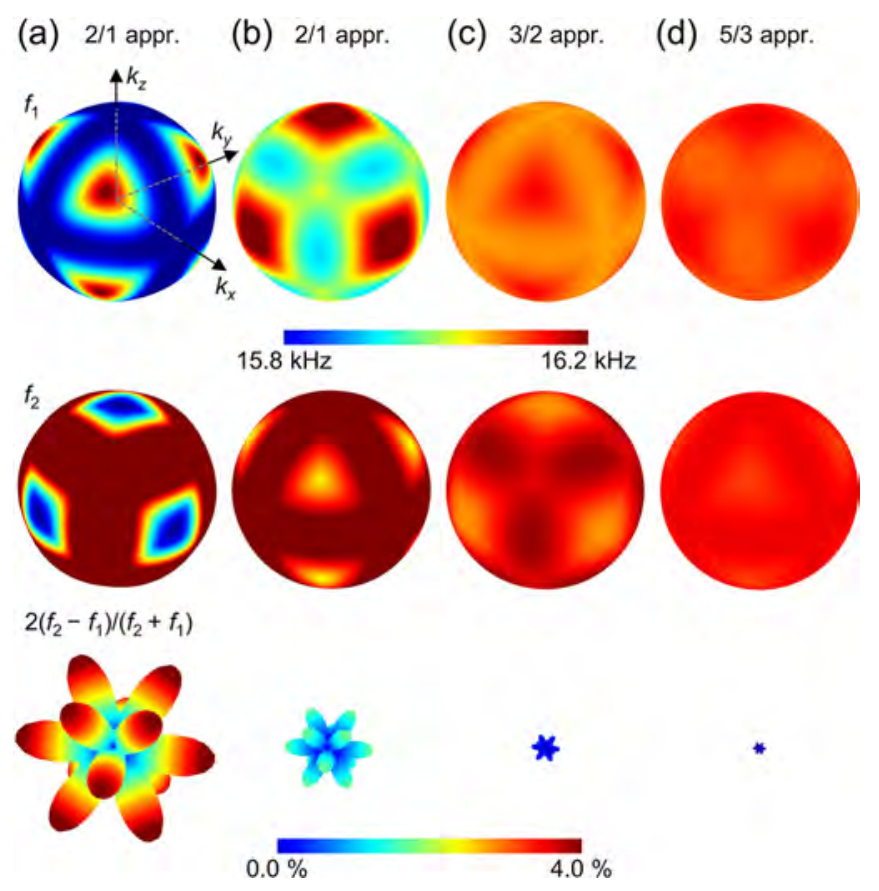

FIG. S4. Calculated direction dependence of the first two transverse eigenfrequencies for the achiral approximants in the long-wavelength limit, $|\mathbf{k}|=1 / 14\left(\pi / a_{6 \mathrm{D}}\right)$. (a) $q / p=1 / 1$, (b) $q / p=2 / 1$, (c) $q / p=3 / 2$, and (d) $q / p=5 / 3$. In the first two rows, we depict the direction dependence of the two transverse phonon eigenfrequencies, $f_{1}$ and $f_{2}$ on a false-color scale. In the third row, we show the relative frequency splitting between the two transverse eigenfrequencies, $2\left(f_{2}-f_{1}\right) /\left(f_{2}+f_{1}\right)$. All of these refer to a fixed modulus of the wave vector $|\mathbf{k}|=1 / 14\left(\pi / a_{6 \mathrm{D}}\right)$. Note that this choice is smaller than the one for the chiral case in the main paper. We choose a smaller wave number here in order to avoid having to deal with the dense set of gray bands in Fig. S3. In the long-wavelength limit, the relative frequency splitting is independent of the wave number anyway. Upon inspection of the lowest row in panels (a)-(d), it becomes obvious that the relative frequency splitting tends to zero for increasing approximant order, indicating an asymptotically achiral isotropic response. Likewise, when going from (a) to (d), the upper two rows show that the eigenfrequencies become isotropic with increasing approximant order as well. 


\section{COMPARISON OF TIMOSHENKO BEAMS AND SOLID MODEL}

All calculations shown in the Letter are based on using the approximation of Timoshenko beams. It is therefore important to evaluate possible errors. For computational reasons, a comparison for the 3D quasi-crystal approximant architectures is not possible. Therefore, we investigate a simpler test case: We use a one-dimensional lattice of chiral elements as shown in Fig. 1(b), implemented by using Floquet-Bloch-periodic bo $\Omega$ ary conditions along the z-axis. To implement the structure shown in Fig. 1(b) as the unit cell for the Timoshenkobeam model, we use about 60 Timoshenko-beam elements with a mesh size smaller than L/10. To mesh the structure shown in Fig. 1(b) as the unit cell for the solid model, we use about 20 thousands tetrahedra with a mesh size smaller than $r / 3$. Results are shown in Fig. S5. For the relative frequency splitting in Fig. S5(b), which is the most important part in the Letter, the agreement between the solid model and the Timoshenko-beam model is quantitative throughout the entire Brillouin zone. For the absolute frequencies, the solid model consistently yields higher frequencies. This behavior is expected because the solid model leads to stiffer joints between the finite-radius rods than the Timoshenko-beam model, which assumes infinitely thin rods everywhere [40].
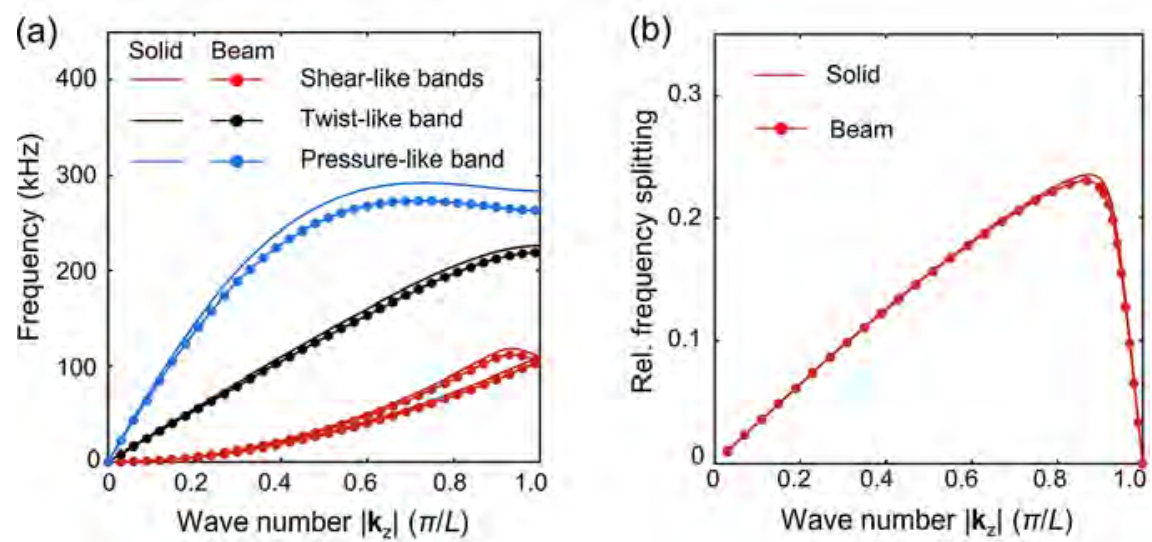

FIG. S5. To check the validity of the approximation in terms of Timoshenko beams, we compare results based on using Timoshenko beams (dots) with corresponding results using a solid model (full curves). (a) Calculated band structure for phonon propagation along the $z$-direction. (b) Relative frequency splitting $2\left(f_{2}-f_{1}\right) /\left(f_{2}+f_{1}\right)$ between the two lowest-frequency shear-like bands, as derived from the data in (a). 


\section{CALCULATED RESULTS FOR CHIRAL APPROXIMANTS}

Chiral approximants of icosahedral quasi-crystal lattices have been obtained by replacing each segment in Fig. 1(a) by a chiral meta-rod. For the chiral meta-rod, we consider the geometric parameters as already quoted in the caption of Fig. $1: d / L=0.3, R / L=0.2$, $h / L=0.035, r / a_{6 \mathrm{D}}=0.01$ (leading to the same rod radius for all rods in the architecture for fixed $a_{6 \mathrm{D}}$ ), and fixed $a_{6 \mathrm{D}}=100 \mu \mathrm{m}$. For all rods, we use the Young's modulus $E=4.18 \mathrm{GPa}$, the Poisson's ratio $\nu=0.4$, and the mass density $\rho=1.15 \times 10^{3} \mathrm{~kg} / \mathrm{m}^{3}$. These material parameters correspond to those of typical polymers, amenable to 3D laser printing [18]. In similar to previous cases, all the rods are modeled using the Timoshenko beams with a mesh size smaller than $L / 10$.

Figure S6 shows the same data as Figs. 2(a)-(d) but in magnified form. Figure S7 exhibits two representative eigenmodes. In Fig. S8, we demonstrate the importance of a large local coordination number by leaving away the connections along the face-diagonals (see blue dotted segments in Fig. 1(a)) and body diagonals (see dashed gray segment in Fig. 1(a)). The result in Fig. S8 can directly be compared with Fig. 4.
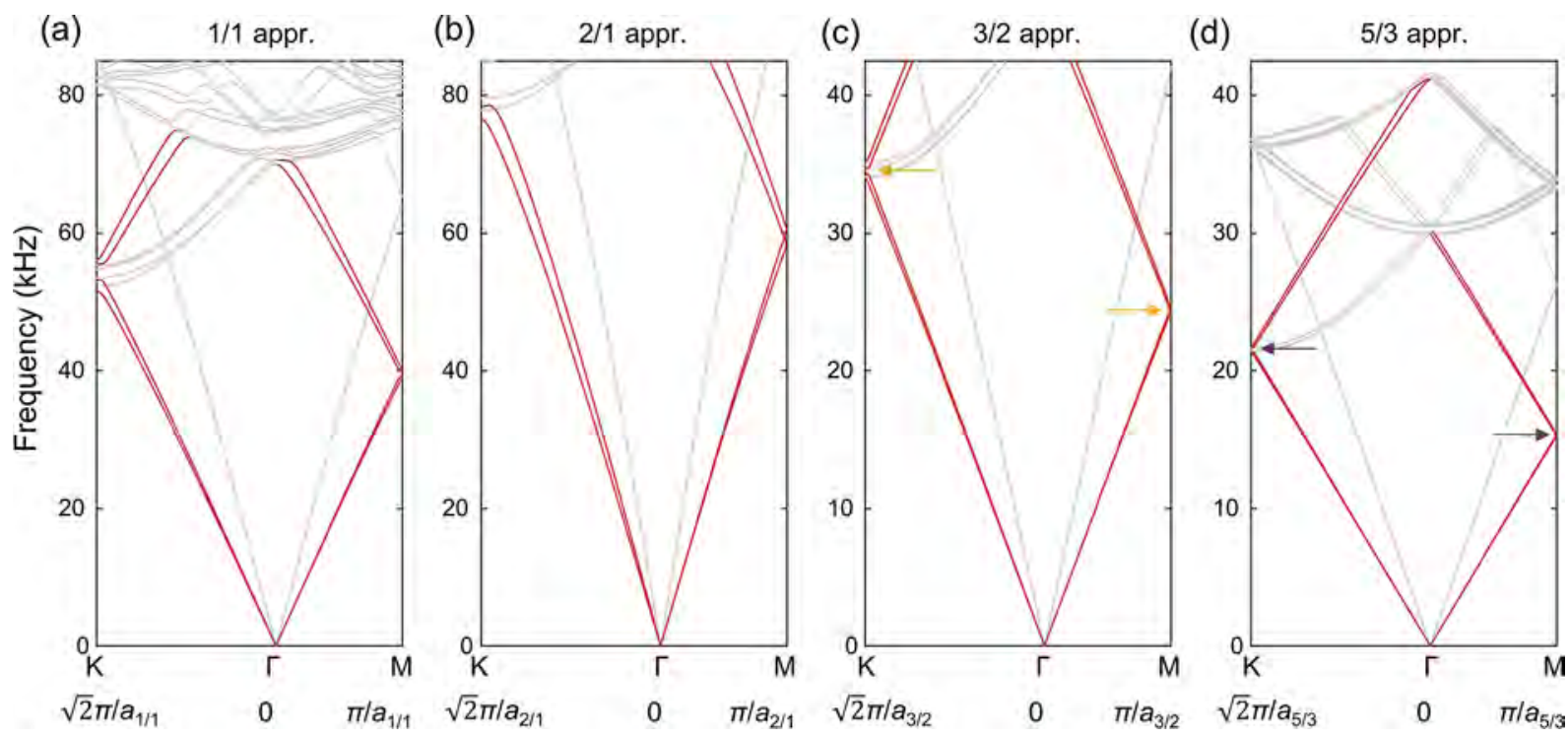

FIG. S6. (a)-(d), same data as in Figs. 2(a)-(d), but in magnified form. 


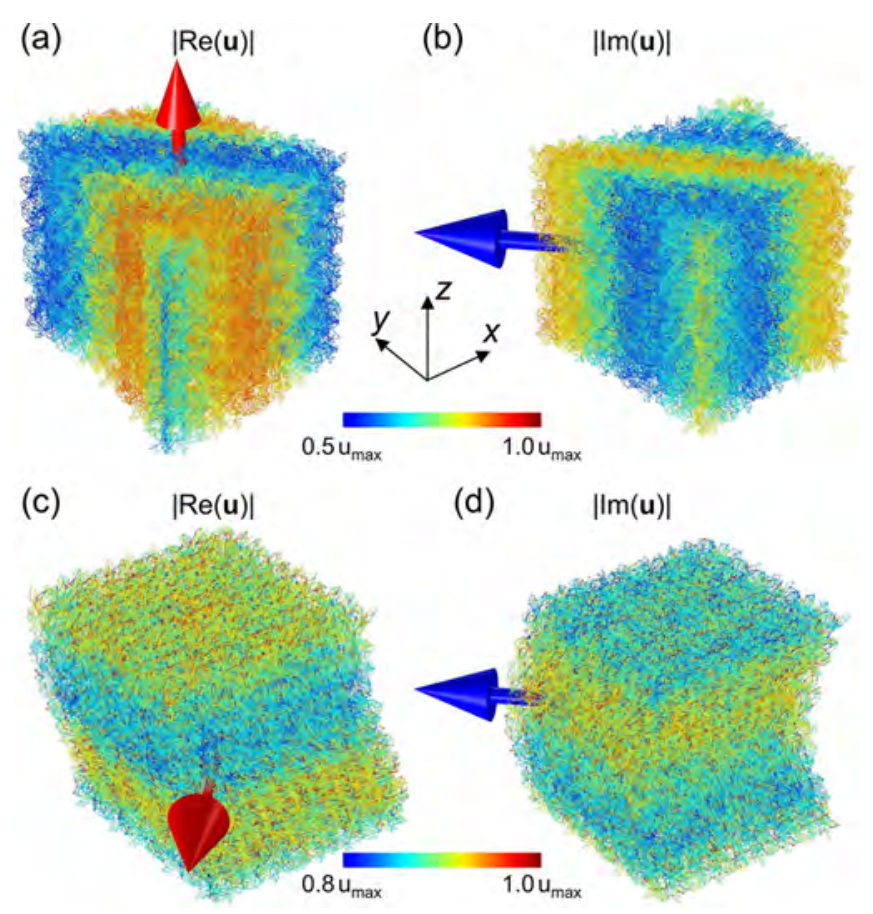

FIG. S7. Representation of the transverse eigenmodes corresponding to the first transverse band for the $q / p=5 / 3$ chiral approximant. For clarity, the displacements have been largely exaggerated. (a) Real and (b) imaginary parts of the displacement-vector field within one unit cell for a wave vector $\mathbf{k}=(1 / \sqrt{2}, 1 / \sqrt{2}, 0) 1 / 9\left(\pi / a_{6 \mathrm{D}}\right)$ along a cubic face diagonal. The red and the blue arrows indicate the real and the imaginary parts of the mean of the displacement vector over one approximant unit cell. The phonon polarization is circular because the red and the blue arrows are orthogonal to each other, and the real and the imaginary parts are phase delayed by 90 degrees. (c) and (d) are analogous to (a) and (b), but for a wave vector $\mathbf{k}=(0,0,1) 1 / 9\left(\pi / a_{6 \mathrm{D}}\right)$ along a principal cubic direction. Results for the two propagation directions are further visualized in Supplemental Video 2 and 3 , respectively. 


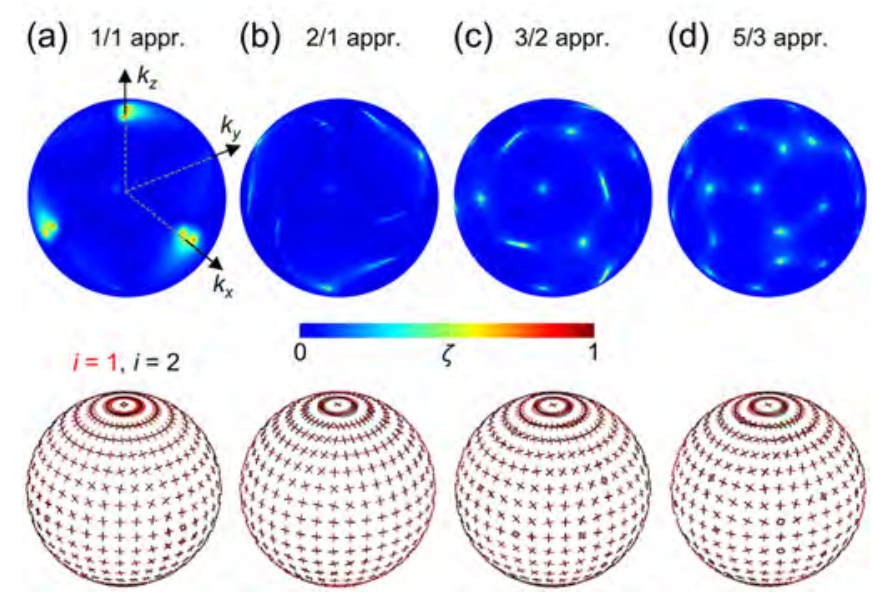

FIG. S8. As Fig. 4, but the connections along the face-diagonals (see blue dotted segments in Fig. 1(a)) and the body diagonals (see dashed gray segment in Fig. 1(a)) have been left away. Therefore, only the red connections in Fig. 1(a) are left. As a result, isotropy is by far not yet reached for the $5 / 3$ approximant (cf. Fig. 4). 
Supplemental Video 1: Animated version of Fig. 1(a).

Supplemental Video 2: Animation of the first transverse eigenmode corresponding to Figs. S7(a) and (b). The red arrow represents the instantaneous displacement vector.

Supplemental Video 3: Animation of the first transverse eigenmode corresponding to Figs. S7(c) and (d). The red arrow represents the instantaneous displacement vector.

Supplemental File 1: *.x_b file of the considered achiral 1/1 3D quasi-crystal approximant (underlying Fig. S2-S3).

Supplemental File 2: ${ }^{*}$.x_b file of the considered achiral 2/1 3D quasi-crystal approximant (underlying Fig. S2-S3).

Supplemental File 3: *.x_b file of the considered achiral 3/2 3D quasi-crystal approximant (underlying Fig. S2-S3).

Supplemental File 4: *.x_b file of the considered ahiral 5/3 3D quasi-crystal approximant (underlying Fig. S2-S3).

Supplemental File 5: *.x_b file of the considered chiral 1/1 3D architecture (underlying Figs. 2-4).

Supplemental File 6: *.x_b file of the considered chiral 2/1 3D architecture (underlying Figs. 2-4).

Supplemental File 7: *.x_b file of the considered chiral 3/2 3D architecture (underlying Figs. 2-4).

Supplemental File 8: *.x_b file of the considered chiral 5/3 3D architecture (underlying Figs. 2-4). 